
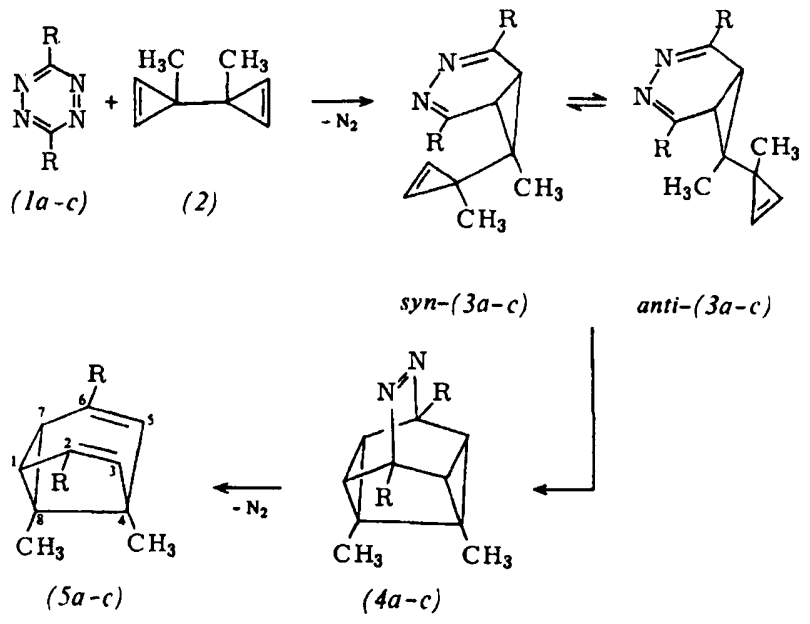

(a), $\mathrm{R}=\mathrm{CO}_{2} \mathrm{CH}_{3} ;$ (b), $\mathrm{R}=\mathrm{C}_{6} \mathrm{H}_{5} ;$ (c), $\mathrm{R}=\mathrm{CH}_{3}$
W. H. De Wolf, I. J. Landheer, F. Bickelhaupt, Tetrahedron Lett. 1975. 179 $J$. Sauer, I. Sellner. H. Sichert, noch unveröffentlicht.

[5] R. Weiss, H. Kölbl, J. Am. Chem. Soc. 97, 3222 (1975) und frühere Arbeiten.

[6] H. E. Zimmerman, H. Iwamura. J. Am. Chem. Soc. 92. 2015 (1970): R. A ska $n i$, Tetrahedron Lett. 197I, 447.

\section{Zusammenhang zwischen Orbitalcharakter und Reaktionsprodukt bei der Umsetzung von Benzvalen und Homobenzvalen mit Thiophenol $\left.{ }^{[*+}\right]$}

Von Manfred Christl, Reinhard Lang, Rainer Herbert und Gertrud Freitag ${ }^{(*)}$

Professor Rolf Huisgen zum 60. Geburtstag gewidmet

Sowohl Olefine ${ }^{[1]}$ als auch Bicyclo[1.1.0]butane ${ }^{[2]}$ addieren Thiole in Radikalkettenreaktionen. Um zu prüfen, welche Funktion der aktivere Partner für das Thiol ist, setzten wir Benzvalen (1) und Homobenzvalen (8), die diese beiden Funktionen enthalten, mit Thiophenol um. Das 1:2-Ge-

Tabelle t. Einige Daten der Semibullvalene (5).

\begin{tabular}{|c|c|c|c|c|c|c|c|c|}
\hline \multirow[t]{2}{*}{ Verb. } & \multirow{2}{*}{$\begin{array}{l}\text { Ausb. } \\
{[\%]}\end{array}$} & \multirow{2}{*}{$\begin{array}{l}\mathrm{Fp}\left[{ }^{\circ} \mathrm{C}\right] \\
\mathrm{Kp}\left[{ }^{\circ} \mathrm{C} / \mathrm{Torr}\right]\end{array}$} & \multicolumn{2}{|c|}{ 'H-NMR [b] } & \multirow[b]{2}{*}{ C-1,3,5.7 } & \multicolumn{2}{|r|}{${ }^{13} \mathrm{C}-\mathrm{NMR}[\mathrm{b}]$} & \multirow[b]{2}{*}{$\mathrm{CH}_{3}$} \\
\hline & & & $H-1,3,5,7$ & $\mathrm{CH}_{3}$ & & $C-4,8$ & C. 2.6 & \\
\hline$(5 a)$ & 48 & $99-100$ & $4.79(s)$ & $1.13(\mathrm{~s})$ & 93.7 (d) & $60.6(s)$ & $127.2(\mathrm{~s})$ & $14.9(q)$ \\
\hline (5b) [6] & 45 & $180 / 0.01$ [a] & $4.36(s)$ & $1.13(\mathrm{~s})$ & 88.7 (d) & $58.5(\mathrm{~s})$ & 133.3 oder $137.3(\mathrm{~s})$ & $15.8(q)$ \\
\hline (5c) [6] & 38 & $40 / 0.01[a]$ & $3.74(\mathrm{~s})$ & $0.98(\mathrm{~s})$ & 90.9 (d) & $59.0(\mathrm{~s})$ & $128.4(s)$ & 15.8 oder $16.3(q)$ \\
\hline
\end{tabular}

[a] Kugelrohrdestillation. [b] In $\mathrm{CDCl}_{3}$, TMS int., $\delta$-Werte: Mittelwertspektren bei Raumtemperatur.

sich erst beim Erwärmen in die Semibullvalene (5b) bzw. (5c) um. Ob ein Gleichgewicht syn-(3) $\rightleftharpoons$ anti-(3) vorliegt, kann nicht entschieden werden. Bei der Reaktion von (1a) mit (2) haben wir (3a) noch nicht nachweisen können; die intramolekulare Addition zu (4a) verläuft offenbar sehr rasch. In keinem Fall konnten Anhaltspunkte für meßbare Konzentrationen von (4) erhalten werden, da (4), durch die beiden anti-ständigen Cyclopropanringe begünstigt, rasch $\mathrm{N}_{2}$ abspaltet und (5) bildet (vgl. ${ }^{[2 a]}$ ).<smiles></smiles>

(6)

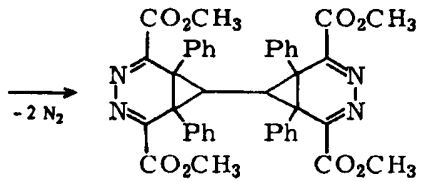

(7)
Die analoge Reaktion mit dem Cyclopropen-Derivat $(6)^{[5]}$ gelang noch nicht. Auch unter Anwendung des Verdünnungsprinzips erhielten wir mit (1a) immer nur das $1: 2$-Addukt (7).

Die von uns erhaltenen Semibullvalene (5) zeigen bei Raumtemperatur bereits die Mittelwertspektren mit ' $\mathrm{H}$ NMR-Signalen für die Methylprotonen von $\delta=1-1.2$, für die Ringprotonen von $\delta=3.7-4.8$. Die analogen ${ }^{13} \mathrm{C}-\mathrm{NMR}$ Mittelwertsignale finden sich für die Methyl-C-Atome um $\delta=15$, für C-4/C-8 bei $\delta=60$ und für C-1, C-3, C-5, C-7 von $\delta=89-94$ (siehe Tabelle 1).

Eingegangen am 3. April 1980 [Z 465]

[1] W. v. E. Doering, W. R. Roth. Tetrahedron 19, 715 (1963)

[2] a) Reichhaltige Literatur findet sich z. B. bei $L$. A. Paquette et al., J. Am. Chem. Soc. 96, 7465, 7474 (1974); 97, 2910 (1975): b) R. Askani, R. Kirslen, B. Dugall, Tetrahedron Lett. 1976, 3891.

[3] G. Heinrichs, H. Krapf, B. Schröder, A. Steigel, T. Troll, J. Sauer, Tetrahedron Lett. 1970, 1617; H. D. Fühlhuber. J. Sauer, ibid. 1977, 4393; H. D. Fühlhuber, C. Gousetis, T. Troll, J. Sauer, ibid. 1978, 3903.

[4] W. H. De Wolf, F. Bickelhaupt, Recl. Trav. Chim. Pays-Bas 90, 150 (1971) W. H. De Wolf. W. Stol, I. J. Landheer, F. Bickelhaupt, ibid. 90,405 (1971); misch aus (1) und Thiophenol ergab nach $24 \mathrm{~h}$ bei $20^{\circ} \mathrm{C}$ vier Produkte: 3-Phenylthiotricyclo[3.1.0.0.6 ${ }^{2.6}$ hexan (2), exo- und endo-4-Phenylthiobicyclo[3.1.0]hex-2-en (6) $(6: 1)$ und 2exo,5-endo-Bis(phenylthio)bicyclo[2.1.1]hexan (3). Konstitution und Stereochemie folgen aus den NMR-Spektren (Tabelle 1).

Tabelle 1. NMR-Daten der neuen Verbindungen (in $\mathrm{CDCl}_{3}, \delta$-Werte, $J$ in $\mathbf{H z}$ )

(2): 'H-NMR: $1.22\left(\right.$ dd. $\left.4-\mathrm{H}_{\mathrm{rtan}} J_{3.4}=3.1, J_{4.4}=11.7\right), 1.83\left(\mathrm{dd} .4-\mathrm{H}_{\text {trun.. }}, J_{3.4}=8.2\right)$, 1.86-1.96 (m, 1-H, 5-H, 6-H), 2.19 (m, 2-H), 3.31 (dd, 3-H), 6.88-7.48 (m, $\left.\mathrm{C}_{6} \mathrm{H}_{5}\right)$ ${ }^{13} \mathrm{C}-\mathrm{NMR}: 3.4$ (d, C-1), 5.8 (d, C-6), 32.9 (d, C-5), 35.5 (t, C-4), 39.3 (d, C-2), 46.3 (d, C-3); $\mathrm{C}_{6} \mathrm{H}_{5}: 125.4$ (d), 128.6 (d), 129.3 (d), 137.5 (s)

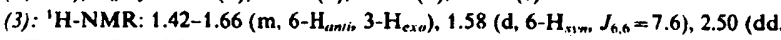
3- $\left.\mathrm{H}_{\text {endin }} \quad J_{2.3}=8.2, \quad J_{3.3}=12.2\right), \quad 2.74 \quad(\mathrm{~m}, \quad 1-\mathrm{H}, \quad 4-\mathrm{H}$ ), 3.53 (q. $5-\mathrm{H}$, $\left.J_{1.5}=J_{3 e \times 0.5}=J_{4.5}=2.4\right), 3.96(\mathrm{~m}, 2-\mathrm{H}), 6.95-7.45\left(\mathrm{~m}, \mathrm{C}_{6} \mathrm{H}_{5}\right)$

exo-(6): 'H-NMR [a]: -0.10 (q. 6- $\left.\mathrm{H}_{\text {ender }} J_{1.6}=J_{5.6}=J_{6.6}=3.9\right), 0.69$ (dt, 6- $\mathrm{H}_{\text {exs }}$ $\left.J_{1.6}=J_{5.6}=7.6\right), 1.40-2.10(\mathrm{~m}, 1 \cdot \mathrm{H}, \mathrm{s}-\mathrm{H}), 4.00(\mathrm{~m}, 4-\mathrm{H}), 5.44\left(\mathrm{~m}, 3-\mathrm{H}, J_{2.3}=5.5\right)$, $6.10(\mathrm{~m}, 2-\mathrm{H}) ; \mathrm{C}_{6} \mathrm{H}_{5}: 7.15-7.50(\mathrm{~m} .3 \mathrm{H})$ und $7.50-7.85(\mathrm{~m}, 2 \mathrm{H}):{ }^{13} \mathrm{C}-\mathrm{NMR}: 19.5$ (t. C-6), 23.4 (d) und 23.8 (d) (C-1 und C-5), 54.3 (d, C-4), 126.7 (d, C-3), 137.4 (d, C-2); $\mathrm{C}_{6} \mathrm{H}_{5}: 126.3$ (d), 128.5 (d), 131.2 (d), 135.1 (s)

(9): 'H-NMR: 1.53 (d, 7- $\mathrm{H}_{\mathrm{v}, \mathrm{m},} J_{7,7}=8.2$ ), 2.00 (dt, 7- $\mathrm{H}_{4 m, i,} J_{1,7}=J_{5,7}=5.4$ ), 2.15 und 2.75 (br. AB-System, 4- $\mathrm{H}_{\text {endis }}$ und 4- $\left.\mathrm{H}_{\text {exmo }} J_{4.4}=18.3\right), 2.65-2.90(\mathrm{~m}, 1-\mathrm{H}, \mathrm{s}$ H), $3.76\left(\mathrm{t}, 6-\mathrm{H}, J_{1.6}=J_{5,6}=5.2\right), 5.69(\mathrm{~m})$ und $6.08(\mathrm{~m})\left(2-\mathrm{H}, 3-\mathrm{H}, J_{2.3}=9.0\right)$ $6.90-7.50\left(\mathrm{~m}, \mathrm{C}_{6} \mathrm{H}_{5}\right)$

[a] In $C_{6} D_{6}$.

Eine mechanistische Studie legt eine Radikalkettenreaktion nahe, wobei sich das Phenylthioradikal, wohl durch Luftsauerstoffoxidation aus Thiophenol entstanden, an die Doppelbindung in (1) addiert. Dem resultierenden Radikal (4) stehen zwei Wege offen: Wasserstoffabstraktion aus

[*] Prof. Dr. M. Christl, Dipl.-Chem. R. Lang, R. Herbert, G. Freitag Institut für Organische Chemie der Universität Am Hubland, D-8700 Würzburg

[**] Vorgetragen auf der Chemiedozententagung 1979 in Darmstadt. Diese Arbeit wurde von der Deutschen Forschungsgemeinschaft und dem Fonds der chemischen Industric unterstützt. 


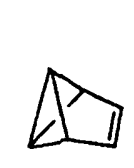

(l)

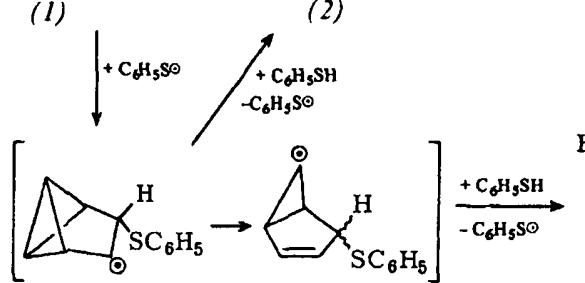

(4)

(5)

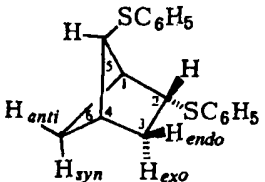

(3)

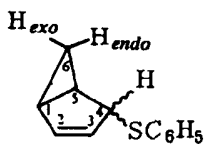

(6)

Thiophenol führt zu (2), vorherige Bindungsumordnung im Sinne einer Cyclopropylmethyl-Homoallyl-Radikalumlagerung $^{[3,4]}$ [zu (5)] ergibt (6). Die Anlagerung von Thiophenol an die zentrale Bicyclo[1.1.0]butan-Bindung von (2) unter Bildung von (3) folgt bekannten Pfaden ${ }^{[2]}$; sie läuft allerdings erheblich langsamer $\mathrm{ab}$ als die Erstaddition (siehe unten).

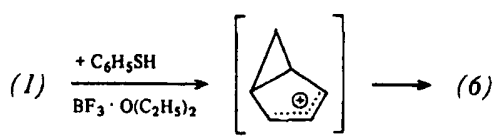

(7)

(6) könnte auch über das Kation (7) entstehen ${ }^{[5]}$. Wir haben diesen ionischen Verlauf durch Zusatz von Bortrifluorid-Ether erzwungen und in $82 \%$ Ausbeute reines (6), $\mathrm{Kp}=60-80^{\circ} \mathrm{C}$ (Bad) $/ 0.01$ Torr, erhalten. Aufgrund von Markierungsexperimenten scheidet dieser Weg aber bei Abwesenheit von Bortrifluorid-Ether aus.

Die von der Zwischenstufe (4) ausgehenden Konkurrenzschritte fordern aufgrund unterschiedlicher Molekularität die Abhängigkeit des (2) : (6)-Verhältnisses von der Thiophenolkonzentration. Dies wurde auch gefunden:

Aus $6.40 \mathrm{mmol}$ (1) und $27.2 \mathrm{mmol}$ Thiophenol in $13 \mathrm{ml}$ Ether bildeten sich in $20 \mathrm{~min}$ bei $20^{\circ} \mathrm{C} 92 \%$ des $96: 4-\mathrm{Gemi}-$ sches aus (2) und (6), $\mathrm{Kp}=60^{\circ} \mathrm{C} / 0.01$ Torr.

Wurden dagegen $6.44 \mathrm{mmol}$ Thiophenol in $50 \mathrm{ml}$ Ether im Laufe von $7 \mathrm{~h} \mathrm{zu} 6.40 \mathrm{mmol}$ (1) in $13 \mathrm{ml}$ Ether getropft, so waren $83 \%$ (2) und (6) im Verhältnis 12:88 zu isolieren.

Wie bei anderen Tricyclo[3.1.0.0 $\left.0^{2.6}\right]$ hexan-Derivaten beobachtet $^{|6|}$, fördert UV-Bestrahlung die Thiophenoladdition auch an (2) beträchtlich. Unter Einwirkung von $350 \mathrm{~nm}$ Licht erhielten wir aus $2.56 \mathrm{mmol}$ (1) und $45.4 \mathrm{mmol}$ Thiophenol in $5 \mathrm{ml}$ Ether nach $18 \mathrm{~h} 87 \%$ (3) in farblosen Kristallen, $\mathrm{Fp}=54-55^{\circ} \mathrm{C}$.

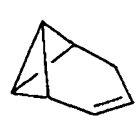

(8)

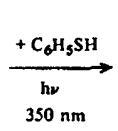

$350 \mathrm{~nm}$

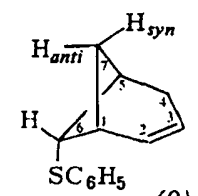

(9)

Homobenzvalen (8) reagiert bei Raumtemperatur nur langsam mit Thiophenol. Bei Bestrahlung mit $350 \mathrm{~nm}$-Licht dagegen läuft die Addition in $2 \mathrm{~h}$ vollständig ab, wobei 6endo-Phenylthiobicyclo[3.1.1]hept-2-en (9) in 73\% Ausbeute ohne Isomere entsteht. Die durch das 'H-NMR-Spektrum (Tabelle 1) bewiesene Struktur sowie die Reaktionsbedingungen sprechen zusammen mit Literaturergebnissen ${ }^{[2.6]}$ für die bei Bicyclo[1.1.0]butanen akzeptierte Radikalkettenreaktion $^{(2)}$.

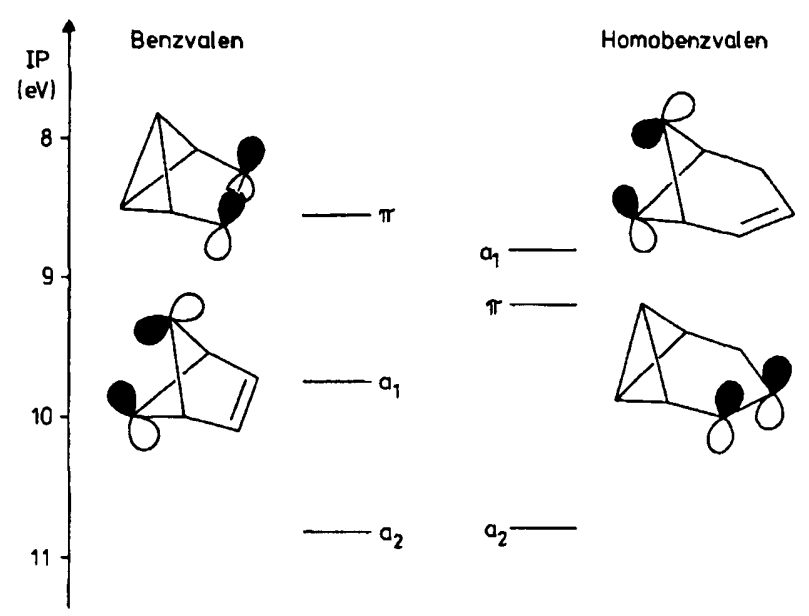

Abb. 1. Jonisationspotentiale und Orbitale von Benzvalen (1) und Homobenzvalen (8) nach [8].

Warum attackiert nun das Phenylthioradikal Benzvalen (1) an der Doppelbindung, Homobenzvalen (8) aber am Bicyclo[1.1.0]butan-System? Da Thioradikale elektrophilen Charakter haben ${ }^{[7]}$, dürfte im Übergangszustand der Addition die Wechselwirkung mit dem HOMO des Substrats entscheidend sein. Wie die photoelektronenspektroskopisch ermittelten und aufgrund von MINDO/3-Rechnungen zugeordneten Ionisationspotentiale zeigen ${ }^{[8]}$, ist das HOMO in (1) in der Doppelbindung, in (8) aber in der Bicyclo[1.1.0]butanZentralbindung lokalisiert (Abb. 1).

Eingegangen am 28. Mai $1979 \quad[\mathrm{Z} 467$ a] Auf Wunsch der Autoren erst jetzt veröffentlicht

CAS-Registry-Nummern

(I): $659-85-8$ / (2): 73688-28-5 / (3): 73688-29-6 / (6)-exo: 73688-30-9 / (6). endo: 73688-31-0 / (8): 35618-58-7 / (9): 73688-32-1 / $\mathrm{C}_{6} \mathrm{H}_{5} \mathrm{SH}:$ 108-98-5.

[1] Ubersicht: K. Griesbaum. Angew. Chem. 82, 276 (1970); Angew. Chem. Int. Ed. Engl. 9, 273 (1970).

[2] G. Szeimies, A. SchloBer, F. Philipp, P. Dietz, W. Mickler, Chem. Ber. III, 1922 (1978).

[3] Übersicht: J. W. Wilt in J. K. Kochi: Free Radicals. Vol. 1. Wiley, New York 1973, S. 333.

[4] Weiteres Beispiel einer Bicyclobutylmethylradikal-Umlagerung: J. Elzinga. H. Hogeveen, J. Org. Chem. 44, 2381 (1979).

[5] L. Kaplan, D. J. Rausch, K. E. Wilzbach. J. Am. Chem. Soc. 94, 8638 (1972), zit. Lit.; J. A. Berson, N. M. Hasty, Jr.. ibid. 93, 1549 (1971); T. J. Katz. E. J. Wang, N. Acton, ibid. 93, 3782 (1971).

[6] R. Herbert, M. Christl, Chem. Ber., 112, 2012 (1979).

[7) C. Walling, D. Seymour, K. B. Wolfstirn, J. Am. Chem. Soc. 70. 2559 (1948).

[8] Benzvalen: P. Bischof, R. Gleiter, E. Müller. Tetrahedron 32. 2769 (1976): P. J. Harman, J. E. Kent, T. H. Gan, J. B. Peel, G. D. Willet, J. Am. Chem. Soc. 99, 943 (1977). Homobenzvalen: P. Bischof, R. Gleiter, R. T. Taylor, A. R Browne, L. A. Paquette, J. Org. Chem. 43, 2391 (1978).

Oxidationsreaktionen am Benzvalen: Ozonolyse, cis-Hydroxylierung, Epoxidation und Singulettsauerstoff-Addition ${ }^{[*]}$

Von Hartmut Leininger und Manfred Christl ${ }^{* 1}$

Professor Rolf Huisgen zum 60. Geburtstag gewidmet

Gute Zugänglichkeit und hohe Reaktivität prädestinieren Benzvalen (1) zu einem nützlichen Edukt für viele Bicyclo[1.1.0]butan-Derivate und andere Kleinring-Polycy-

[*] Prof. Dr. M. Christl. Dipl.-Chem. H. Leininger Institut für Organische Chemie der Universität Am Hubland. D-8700 Würzburg

1**] Diese Arbeit wurde von der Deutschen Forschungsgemeinschaf und dem Fonds der Chemischen Industrie unterstützt. 